\section{Determining Sex and Screening for the Adventitious Presence of Transgenic Material in Carica papaya L. Seed Germplasm}

\author{
Tracie K. Matsumoto ${ }^{1}$, Francis T.P. Zee, Jon Y. Suzuki, \\ Savarni Tripathi, and James Carr \\ USDA, ARS, Pacific Basin Agricultural Research Center, Tropical Plant \\ Genetic Resource and Disease Research Unit, P.O. Box 4459, Hilo, HI 96720
}

Bruce Mackey

USDA, ARS, Pacific West Area, 800 Buchanan Street, Albany, CA 94710

Additional index words. hermaphrodite, female, polymerase chain reaction (PCR), Papaya ringspot virus (PRSV), GUS, papain, GMO detection

\begin{abstract}
Papaya ringspot virus (PRSV) is a devastating disease that has a detrimental impact on both commercial papaya production and Caricaceae germplasm conservation. In 1998, the PRSV coat protein transgenic line 55-1 and derived progeny were released to growers in Hawaii. The transgenic varieties have provided durable and practical control of the disease that have saved the papaya industry. However, like with transgenic crops throughout the world, there is public concern about the possibility of cross-contamination of these transgenic materials into nontransgenic lines. As the designated germplasm repository for Caricaceae, we are responsible for maintaining the genetic integrity of each accession. Therefore, we have developed a protocol using polymerase chain reaction for detection of the adventitious presence of the 55-1 transgene insertion event in both parental plants and their progeny seed populations. This protocol assures a $99.9 \%$ confidence level of obtaining seeds that are $\mathbf{9 9 . 5 \%}$ transgene-free. The protocol developed in this study is not typical for most seed validation techniques because there is a higher than normal producer risk resulting from the potential of large numbers of seeds not meeting the stringent criteria. However, we believe this is necessary to ensure the genetic integrity of seeds stored in the repository.
\end{abstract}

The Caricaceae family consists of six genera, including Vasconcellea, which contains 21 of the 35 Caricaceae species, and Carica papaya L., which is the most economically important species attributable largely to its cultivation in the tropics for fruit production (Van Droogenbroeck et al., 2004). Breeding and selection of C. papaya for cultivation has resulted in the development of numerous C. papaya varieties and this comprises the majority of accessions at the USDA, ARS, PBARC, Tropical Plant Genetic Resources and Disease Research (TPGRDR) unit in Hilo, HI. The TPGRDR is part of the National Plant Germplasm System and the designated location for Caricaceae germplasm.

Received for publication 17 Aug. 2009. Accepted for publication 7 Nov. 2009.

We thank Russell Kai, Mariel Mogote, Angelica Tangalin, and Tsuyoshi Tsumura for their excellent technical assistance.

Mention of a trademark, proprietary product, or vendor does not constitute a guarantee or warranty of the product by the U.S. Department of Agriculture and does not imply its approval to the exclusion of other products or vendors that also may be suitable.

${ }^{1}$ To whom reprint requests should be addressed; e-mail tracie.matsumoto@ars.usda.gov.
Papaya ringspot virus (PRSV) is a devastating disease that has a detrimental impact in Hawaii on both commercial production and germplasm conservation. Although tolerance but not genetic resistance to PRSV has been reported in the Carica germplasm, PRSVresistant plants have been achieved by genetic engineering with the commercial release of transgenic papaya cultivars SunUp and Rainbow. In 2009, the Hawaii papaya industry is based on 'Rainbow' (77\%), 'Kapoho' (9\%), 'Sunrise' (9\%), and other (5\%) (USDA, National Agricultural Statistics Service and State of Hawaii Department of Agriculture Agricultural Development Division, 2009). The 'SunUp' variety is the homozygous version of the original transgenic line 55-1 and 'Rainbow' is the F1 hybrid cross between 'SunUp' and 'Kapoho' (reviewed in Gonsalves et al., 2006). The plasmid used in the generation of the transgenic line 55-1 consisted of the Agrobacterium transformation vector pGA482GG/ cpPRV4, which contained 70 bases of the 5' Cucumber mosaic virus untranslated region fused to the 16 amino acid sequence of the N-terminal end of the Cauliflower mosaic virus coat protein that is translationally fused to the coat protein of PRSV HA 5-1. Expression is controlled by the $35 \mathrm{~S}$ promoter and selection was based on the NPTII (neomy- cinphosphotransferase for kanamycin resistance) and uidA [ $\beta$-glucoronidase (GUS)] reporter genes NCBI accession FJ467933 (Fitch et al., 1992; Ling et al., 1991).

The presence of transgenic papaya plants in Hawaii presents a potential increased risk of contamination of nontransgenic seed sources by the transgenic material. Although there is an ongoing effort to obtain regulatory clearance in Japan for the importation of the transgenic 55-1 papaya, genetically engineered papayas cannot be shipped into Japan or the European Union (Ohmori et al., 2008). Contamination of transgenic material or any other unintentional genetic outcrossing of the germplasm material is of great concern to the USDA TPGRDR because we distribute Caricaceae germplasm to locations throughout the world. Therefore, to optimize our production, we developed a polymerase chain reaction (PCR) protocol to test our material for the presence of the transgene and to determine the sex of the seedlings planted into the field to reduce the labor required to remove the undesired female plants after flowering.

Standard operating procedures at the TPGRDR unit for Caricaceae seed preservation include regeneration of seeds every 4 to 6 years depending on the storage viability of each accession. Papaya seedlings are germinated in the same area that they are planted to reduce the chance of introducing PRSV and other diseases into a new growing area. An average of three to five seedlings (depending on the cultivar) are planted per planting hole to ensure the presence of at least one hermaphrodite plant for gynodioecious accessions or one male or one female plant for dioecious accessions. Once flowering occurs, the remaining female or male plants are removed. Because the hermaphrodite papayas are in-bred, seed production is achieved by bagging flowers in glassine envelopes before anthesis to prevent outcrossing. For highly heterozygous, dioecious papaya lines, it is necessary to gather pollen from all male trees within the same genetic line to pollinate all the female plants to ensure preservation of the genetic integrity of the accession. During the regeneration cycle, observations on plant stature and growth characteristics are monitored to ensure the consistent phenotypic traits for the genetic line. In addition, papaya fruit morphological data are obtained, submitted, and stored in the national plant database system known as the Germplasm Resources Information Network (GRIN). Morphological data on papaya fruit can be obtained at http://www.ars-grin.gov/cgi-bin/ npgs/html/desclist.pl?126.

Because genetically engineered plants are indistinguishable from the original genetic source with the exception of the transgene, it is almost impossible to distinguish the transgenic and nontransgenic material based only on morphological characteristics. Thus, various researchers have used PCR techniques to detect the presence of the transgene insert in 55-1 papaya lines in seeds, seedlings, and fruits (Ohmori et al., 2008; Wall et al., 2004). Identification of the transgene inserts from 
seeds was based on a homogenous population and not a mixture of seeds from transgenic and nontransgenic sources (Wall et al., 2004). Numerous methods have been developed to screen seeds such as maize, canola, soybeans, safflower, and rice for the adventitious presence of transgenes through various PCR techniques for selectable makers, promoters, or genes of interest (Christianson et al., 2008; Demeke et al., 2006; Freese et al., 2007). Statistical methods (i.e., SeedCalc) have been developed to assist in designing and implementing seed testing procedures to detect the adventitious presence of transgenes and analyze risk considerations for seed producers and seed consumers (Remund et al., 2001). We describe a procedure that has a higher than normal producer risk resulting from the potential of large numbers of seeds not meeting the stringent criteria. We believe this is necessary for our role in germplasm conservation and distribution. However, others can still use the same protocols outlined in this article by lowering the stringency of the rejection criteria. To the best of our knowledge, this is the first publication on the detection of the adventitious presence of transgenes in large-scale nonhomogeneous papaya seeds.

\section{Materials and Methods}

Papaya cultivation. Transgenic papaya seeds of 'SunUp' and 'Rainbow' [kindly provided by the Hawaii Papaya Industry Association (HPIA)] and nontransgenic 'Kapoho', 'Sunrise', 'Sunset', 'Waimanalo', and 'Sunrise' $\times$ 'Kapoho' hybrid seeds (provided by TPGRMU and HPIA) were used in the study. Papaya seeds were soaked overnight in water, rinsed three times, soaked for $30 \mathrm{~min}$ in 2.5 $\mathrm{mm} \mathrm{GA}_{3}$ (Dr. Rod. Drew, personal communication), and planted in a 1:1 perlite:vermiculite media in a seedling tray on a heat mat (30 ${ }^{\circ} \mathrm{C}$ ). Misting intervals were set so temperatures did not exceed $30^{\circ} \mathrm{C}$. Seeds generally germinated in 1 to 4 weeks. Seedlings were transplanted at the four true leaf stage to cell packs $\left(256 \mathrm{~cm}^{3}\right)$ consisting of two parts of Sunshine mix \#4 (Sun Gro Horticulture, Bellvue, WA) supplemented with one part of perlite. Plants were kept in a covered greenhouse and fertilized bimonthly with $14.7 \mathrm{~mL}$ of $16 \mathrm{~N}-7 \mathrm{P}-$ $13.3 \mathrm{~K}$ plus micronutrients and monthly with $14.7 \mathrm{~mL} \cdot \mathrm{L}^{-1}$ of $30 \mathrm{~N}-4.4 \mathrm{P}-8.4 \mathrm{~K}$ foliar fertilizer. Plants were field planted 3 to 4 months after initial transplant.

DNA extraction from parental papaya lines. Samples were collected using a No. 8 cork borer. Seven leaf discs $(\approx 100 \mathrm{mg})$ were placed into a $1.5-\mathrm{mL}$ microcentrifuge tube with a hole in the cover to allow air to escape when freezing in liquid nitrogen. The cork borer was washed in water and $70 \%$ ethanol and wiped dry between samples. The plant tissue was frozen in liquid nitrogen and homogenized using a pestle for $1.5-\mathrm{mL}$ microcentrifuge tubes. DNA was extracted using the method from Doyle and Doyle (1990) with minor modifications. Briefly, $100 \mathrm{mg}$ of ground papaya leaf tissue was mixed with a $750-\mu \mathrm{L}$ CTAB solution $(3 \%$
CTAB, 1.4 M NaCl, 100 mm Tris pH 8.0, 20 mM EDTA $\mathrm{pH} 8.0$, and $0.1 \% \beta$-mercaptoethanol), incubated at $60{ }^{\circ} \mathrm{C}$ for $30 \mathrm{~min}$ with shaking, extracted with $750 \mu \mathrm{L}$ chloroform: octanol (24:1), and precipitated with twothirds volume of isopropanol. The DNA pellet was dissolved in $200 \mu \mathrm{L}$ TE buffer, incubated with $1 \mu \mathrm{g} \cdot \mathrm{mL}^{-1} \mathrm{RNase}$ at $37^{\circ} \mathrm{C}$ for $30 \mathrm{~min}$, extracted with phenol:chloroform: isoamyl alcohol (25:24:1), and precipitated with $1 / 10$ volume of $5 \mathrm{M}$ ammonium acetate and two volumes of ethanol. DNA was resuspended in $100 \mu \mathrm{L}$ TE buffer and typically $5 \mu \mathrm{L}$ was used in each PCR reaction.

DNA extraction from papaya seeds. Papaya seeds were counted, placed into a Waring blender (Waring Laboratory and Science, Torrington, CT), and ground with four 10-s bursts with sample mixing between each grinding interval. CTAB buffer preheated to $60{ }^{\circ} \mathrm{C}$ was added to the papaya seed powder and ground for an additional $5 \mathrm{~s}$. The samples were placed in a $250-\mathrm{mL}$ centrifuge tube with $100 \mathrm{~mL}$ CTAB buffer at a final dilution of 100 $\mathrm{mL}$ per 500 seeds. Samples were incubated at $60{ }^{\circ} \mathrm{C}$ for $30 \mathrm{~min}$ with intermittent shaking. The slurry was filtered through Miracloth (Calbiochem, San Diego, CA) and $45 \mathrm{~mL}$ of the filtrate was centrifuged at $4500 \mathrm{rpm}$ in a Marathon 2100R (Fisher Scientific, Pittsburgh, PA) for $10 \mathrm{~min}$ at $4{ }^{\circ} \mathrm{C}$. Thirty milliliters of the resulting supernatant was extracted with $20 \mathrm{~mL}$ of $24: 1$ chloroform:octanol and centrifuged at $4500 \mathrm{rpm}$ for $10 \mathrm{~min}$ at $4{ }^{\circ} \mathrm{C}$. The aqueous layer was removed and precipitated with two-thirds volume of isopropanol. The resulting DNA pellet was washed with $70 \%$ ethanol and air-dried. The DNA was resuspended in $200 \mu \mathrm{L}$ of TE buffer. A DNA cleanup procedure using the Plant DNAeasy kit (Qiagen, Valencia, CA) was used to purify the papaya DNA in which $70 \mu \mathrm{L}$ of the papaya DNA was mixed with $400 \mu \mathrm{L}$ of AP1 buffer. Subsequent steps were followed using the manufacturer's protocol.

Polymerase chain reaction determination of sex and presence of 55-1 transgene insertion. The random amplified polymorphic DNA markers SCAR T1 and SCAR W11 were used to distinguish female and hermaphrodite papaya seedlings (Deputy et al., 2002). The primer sequences used to amplify SCAR T1 and W11 were T1 FOR 5'-TGC TCT TGA TAT GCT CTC TG-3', T1 REV 5'TAC CTT CGC TCA CCT CTG CA-3', W11 FOR 5'-CTG ATG CGT GTG TGG CTC TA-3', and W11 REV 5'-CTG ATG CGT GAT CAT CTA CT-3'

PCR amplification of the transgenic event used the sequence flanking the transgene insertion into the genomic DNA of the papaya plant where the forward primer of the left flanking region (LFR) and the reverse primer of the right flanking region (RFR) are located in papaya genomic DNA outside of the transformed plasmid insertion and the remaining primers are sequences within the transgene (Suzuki and Tripathi, unpublished results). The primer were developed by Dr. Jon Y. Suzuki and Dr. Savarni Tripathi; the sequences are: P55 LFR FOR 5'-ATT TAC
CGC TCC CCG TCT TA-3' ${ }^{\prime}$, P55 LFR REV 5' GGA ACG AAA ACT CAC GTT AAG GG-3', P55 LFR int REV 5' - TTC TTG AAG CAT CTA AGG CTG C-3', P55 RB FOR 5' -CAA AGT TCC GTC ACA GGA TGA T-3', P55 RB REV 5'-TAG TAG GTT TCC ATA CCA AGG CTC-3', and P55 RBint FOR 5' -CCT CCG AAA TAC CAA TAG ACA TGA-3'.

Typically, $5 \mu \mathrm{L}$ of isolated DNA was used for each PCR reaction consisting of $1 \times$ Taq buffer, $2.5 \mathrm{~mm} \mathrm{MgCl}_{2}, 0.2 \mathrm{~mm}$ dNTP, $0.2 \mu \mathrm{M}$ of each primer (with the exception of P55 LFR FOR and P55 RFR REV, which were used at $0.4 \mu \mathrm{M})$, and 1 unit of Taq or Go Taq polymerase (Promega Corp., Madison, WI). The PCR reactions consisted of incubation at $95{ }^{\circ} \mathrm{C}$ for $5 \mathrm{~min}$ followed by 30 cycles of $95^{\circ} \mathrm{C}$ for $30 \mathrm{~s}, 54^{\circ} \mathrm{C}$ for $30 \mathrm{~s}, 72^{\circ} \mathrm{C}$ for $1 \mathrm{~min}$, $30 \mathrm{~s}$ with $5 \mathrm{~s} /$ cycle extension, and then a final extension at $72{ }^{\circ} \mathrm{C}$ for $10 \mathrm{~min}$. PCR samples were run on a $1 \%$ agarose TAE gel.

Polymerase chain reaction detection of $\beta$-glucoronidase and PRSVcp in 55-1 transgene insertion. The multiplex PCR protocol to amplify the GUS (uidA) or PRSVcp within the transgene of 55-1 to indicate the transgenic event and papain as an internal control was used in this study (Wall et al., 2004). The primer sequences are: Papain 1-5 FOR 5'-GGG CAT TCT CAG CTG TTG TA-3', Papain 1-3 REV 5' -CGA CAA TAA CGT TGC ACT CC-3', p35S-cf3 FOR 5' CCA CGT CTT CAA AGC AAG TGG-3', PRSVcp REV 5' -GCA TCC ACA GCT TCA TTC TTG-3', and GUS n $3^{\prime}$-TCG TTA AAA CTG CCT GGC AC-3'.

Typically, $200 \mathrm{ng}$ of purified DNA was used for each PCR reaction consisting of $1 \times$ Taq buffer, $2.5 \mathrm{~mm} \mathrm{MgCl}_{2}, 0.2 \mathrm{~mm} \mathrm{dNTP}, 0.2$ $\mu \mathrm{M}$ of each primer, and 1 unit of Taq or Go Taq polymerase (Promega Corp.). The PCR reaction consisted of incubation at $95^{\circ} \mathrm{C}$ for 5 min followed by 30 cycles of $95^{\circ} \mathrm{C}$ for $30 \mathrm{~s}$, $59^{\circ} \mathrm{C}$ for $25 \mathrm{~s}, 72^{\circ} \mathrm{C}$ for $15 \mathrm{~s}$, and then a final extension at $72{ }^{\circ} \mathrm{C}$ for $5 \mathrm{~min}$ (Wall et al., 2004). PCR samples were run on a $2 \%$ agarose TAE gel.

\section{Results and Discussion}

We describe a protocol to screen the regenerated plant material for the presence of the transgene derived from the original PRSV coat protein transformed line 55-1. We screened the parental line for the presence of the transgene using the left and right flanking regions of the transgene. In addition, we also screened these lines for sex determination to identify the hermaphrodites at an early stage of development. Identification of the hermaphrodites at the seedling stage saves on manpower required to remove the undesired female plants after flowering. Finally, we also describe a procedure to test the resulting seeds for the presence of adventitious transgene insertions in seed material using the genes in the PRSV coat protein gene. We used two different gene products for testing the parental and seed material to accommodate for any rearrangements that may occur through sexual reproduction. 


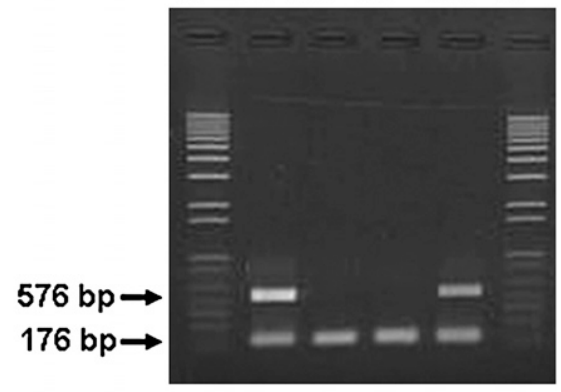

B

RFR

$M$ SU SR KP RB M
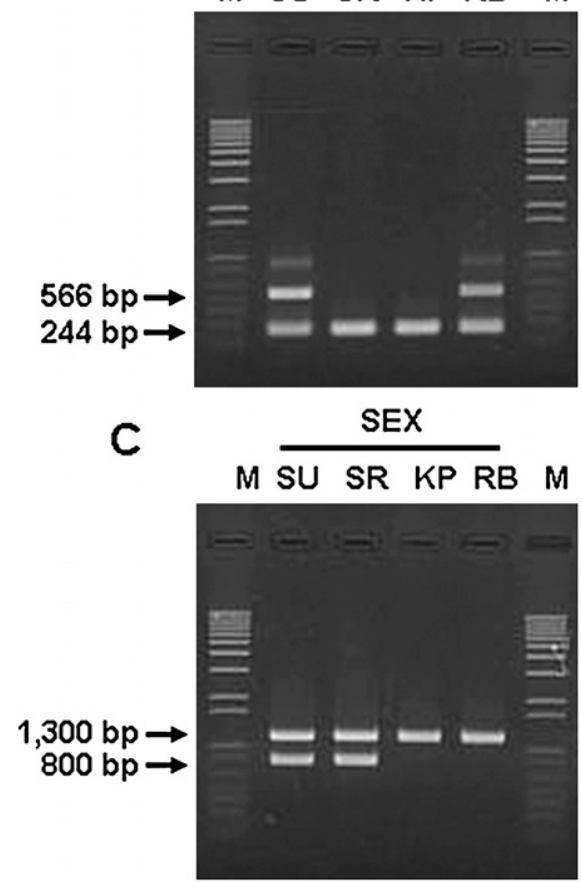

Fig. 1. Polymerase chain reaction amplification of genomic DNA from 'SunUp' (SU), 'Sunrise' (SR), 'Kapoho' (KP), and 'Rainbow' (RB). (A) The higher molecular weight band (576 bp) amplified from a region internal to the transgene and in the left flanking region (LFR) genomic DNA and present only in transgenic lines ( $\mathrm{SU}$ and $\mathrm{RB}$ ), whereas the lower molecular weight (176 bp) band is external to the transgene and present in both transgenic (SU and $\mathrm{RB}$ ) and nontransgenic (SR and $\mathrm{KP}$ ) papaya lines. (B) The higher molecular weight band (566 bp) amplified from a region internal to the transgene and in the right flanking region (RFR) genomic DNA and present only in transgenic lines (SU and $\mathrm{RB}$ ), whereas the lower molecular weight (244 bp) band is external to the transgene and present in both transgenic (SU and RB) and nontransgenic (SR and KP) papaya lines. (C) SCAR T1 (1300 bp) and SCAR W11 (800 bp) were used to determine the sex of the papaya lines where the presence of both bands indicated the hermaphrodites (SU and SR), whereas the amplification of the $\mathrm{T} 1$ band alone indicated female lines (KP and RB) (Deputy et al., 2002).

Polymerase chain reaction determination of sex and presence of 55-1 transgene insertion event in parental lines. We have tested over 1000 papaya seedlings of
'Kapoho', 'Rainbow', 'Sunrise', 'SunUp', and 'Waimanalo' for sex determination with an accuracy of $\approx 90 \%$. Although this is lower than the published accuracy of 99\% (Deputy et al., 2002), we believe the error rate may not be the result of PCR detection, but instead inadvertent mixing of material at the time of transplanting from the cell pack to the field. We now have a revised protocol in which three to five seedlings are planted into the field and individual plants are flagged and tested by PCR. If the plant is found to be female, the seedling is removed and the next seedling is then flagged and tested by PCR. This process is continued until the entire field contains one hermaphrodite plant per hole.

We have tested over 300 seedlings of nontransgenic papaya accessions 'Kapoho', 'Sunrise', and 'Waimanalo' with the LFR primer pair and we have not found the presence of the transgene insertion. Seedlings used in this study were generated from seeds produced within the last 2 to 3 years during a period in which 'Rainbow' was the most common papaya cultivar grown in Hawaii. This is an encouraging indicator that the current methods to exclude extraneous pollen from our germplasm material are currently working. We believe the absence of transgenic material from our germplasm material is a result of the bagging of the flower before the stigma is receptive to pollen to prevent unexpected contamination. Although this added PCR procedure may not be practical for testing all germplasm material, the relatively small number of Carica papaya accessions, compared with other seed crops such as maize, makes it feasible to test our material. An example of a gel of papaya seedlings tested for sex and the presence of the transgene is shown in Figure 1.

Polymerase chain reaction detection of $\beta$-glucoronidase and PRSVcp in papaya seeds. To test each batch of seeds, we determined that a zero tolerance testing plan of SeedCalc Version 7.1 (Remund et al., 2001), which uses 1500 seeds, will result in a $99.9 \%$ confidence level that the tested seeds will be $99.54 \%$ transgene-free. These calculations are further supported by the calculated statistics in Couey and Chew (1986). To determine the limits of detection for our system, we used transgenic:nontransgenic seeds at ratios of $0: 500,1: 100 ; 1: 250,1: 500 ; 1: 1000$, and 1:2000 (Fig. 2). Although the presence of the transgenes could be detected in concentrations as low as 1:2000, we took a conservative approach and used three random samples of 500 seeds to test a total 1500 seeds. In all of our routine testing procedures, in parallel to our samples, we extracted DNA from previously tested nontransgenic seed batches to serve as negative controls and DNA extracted from a 1:500 transgenic seed to nontransgenic seeds to serve as a positive PCR amplification control.

In our seed testing procedure, we tested for the presence of GUS (uidA) and PRSV coat protein segments (Wall et al., 2004). We used these primer sets for two reasons; the

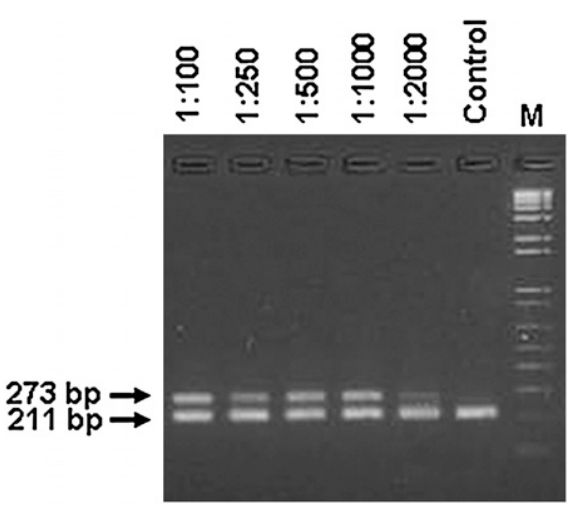

Fig. 2. PCR amplification of genomic DNA from nontransgenic seeds "spiked" with seeds from 'SunUp' at concentrations of 1:100, 1:250, $1: 500,1: 1000,1: 2000$, and control (0:500 nontransgenic) seeds. The papain segment $(211 \mathrm{bp})$ is amplified in all samples, whereas the PRSVcp (273 bp) segment is only present in the seed lots "spiked" with transgenic seeds. Transgene detection can be observed at concentrations as low as 1:2000.

first is that these primers have been established to work in papaya seeds (Wall et al., 2004) and second is that these primers amplify different segments than those used for analysis of the parental lines. These primers would still detect the transgene in case of subsequent rearrangements of the genomic DNA. In addition, because the GUS gene is a common transformation marker, these primers would be able to detect transgenic papaya seeds derived from other transformation events that are mixed into the seed population. As more transgenic papaya lines are released in the United States, we will modify these primers to ensure that the seeds will be tested for all of the different transgenic events.

From these results, we have generated a working protocol for the regeneration of Carica papaya seed. Papaya seeds are either directly seeded in the field or three to five seedlings are transplanted per planting space. DNA is extracted from the leaves of the papaya plants and is subjected to two PCR protocols, the first to determine the sex of the plant using the SCAR W11 and SCAR T1 primer sets (Deputy et al., 2002) and second to determine the presence or absence of the 55-1 transgenic event using the 55-1 LFR and/or RFR primer sets (Suzuki and Tripathi, unpublished results). Female plants and transgenic plants would be discarded and the screening process would continue until hermaphrodite and nontransgenic plant material is achieved. Descriptor data of the growth characteristics of the plant, flower, and fruits will be noted to ensure these data are consistent within the accession. Once the papaya plant matures to flowering, the flowers are bagged before anthesis with glassine envelopes and tagged with the genetic cross and date. As the fruit matures, the information on the tag is written directly onto the fruit. The fruits are harvested at color break and seeds of the same genetic cross are processed together. The seeds are cleaned to remove 
the sarcotesta using a food processor with plastic blades and dried in a dehumidifier cabinet to a moisture content of $\approx 5 \%$. Three random samples of 500 seeds are taken for seed testing using the uidA and PRSVcp primer sets (Wall et al., 2004). Seeds will be tested with negative and positive controls and if determined to contain the transgene, a second sample will be tested. If the presence of the transgene is confirmed, the seed lot will be destroyed. In addition to testing for the adventitious presence of the 55-1 transgene construct, 100 seeds are used to determine the germination percentage and $100 \mathrm{~g}$ of seed is placed into storage. We believe that our current management method, together with testing the parental lines for the presence of the transgene, will further ensure the genetic integrity of our Carica papaya germplasm accessions.

\section{Literature Cited}

Christianson, J., M. McPherson, D. Topinka, L. Hall, and A.G. Good. 2008. Detecting and quantifying the adventitious presence of transgenic seeds in safflower, Carthamus tinctorius L. J. Agr. Food Chem. 56:5506-5513.

Couey, H.M. and V. Chew. 1986. Confidence limits and sample size in quarantine research. J. Econ. Ent. 79:887-890.

Demeke, T., D.J. Perry, and W.R. Scowcroft. 2006. Adventitious presence of GMOs: Scientific overview for Canadian grains. Can. J. Plant Sci. 86:1-23.

Deputy, J.C., R. Ming, H. Ma, Z. Liu, M.M.M. Fitch, M. Wang, R. Manshardt, and J.I. Stiles. 2002. Molecular markers for sex determination in papaya (Carica papaya L.). Theor. Appl. Genet. 106:107-111.

Doyle, J.J. and J.L. Doyle. 1990. Isolation of plant DNA from fresh tissue. Focus 12:1315.

Fitch, M.M.M., R.M. Manshardt, D. Gonsalves, J.L. Slightom, and J.C. Sanford. 1992. Virus resistant papaya plants derived from tissues bombarded with the coat protein gene of papaya ringspot virus. BioTechnology 10: $1466-1472$.

Freese, L., T.A. Scholdberg, D.D. Burton, T.D. Norden, L.A. Shokere, and G.R. Jenkins. 2007. Evaluating homogeneity of LL601 rice in commercial lots using quantitative realtime PCR. J. Agr. Food Chem. 55:60606066.

Gonsalves, D., A. Vegas, V. Prasartsee, R. Drew, J.Y. Suzuki, and S. Tripathi. 2006. Developing papaya to control papaya ringspot virus by transgenic resistance, intergeneric hybridization, and tolerance breeding. Plant Breed. Rev. 26:35-78

Ling, K., S. Namba, C. Gonsalves, J.L. Slightom, and D. Gonsalves. 1991. Protection against detrimental effects of potyvirus infection in transgenic tobacco plants expressing the papaya ringspot virus coat protein gene. BioTechnology 9:752-758.
Ohmori, K., J. Tsuchiya, T. Watanabe, H. Akiyama, T. Maitani, T. Yamada, K. Hirayama, and S. Satoh. 2008. A DNA extraction method using silica-base resin type kit for the detection of genetically modified papaya. J. Food Hyg. Soc. Jpn. 49:63-69.

Remund, K.M., D.A. Dixon, D.L. Wright, and L.R. Holden. 2001. Statistical considerations in seed purity testing for transgenic traits. Seed Sci. Res. 11:101-119.

USDA, ARS, National Genetic Resources Program. Germplasm Resources Information Network (GRIN) [online database]. National Germplasm Resources Laboratory, Beltsville, MD. 16 Sept. 2008. <http://www.ars-grin.gov/ cgi-bin/npgs/html/desclist.pl?126>.

USDA, National Agricultural Statistics Service and State of Hawaii Department of Agricutlure Agricultural Development Division. Hawaii papayas. 27 Oct. 2009. <http://www.nass.usda. gov/Statistics_by_State/Hawaii/Publications/ Fruits_and_Nuts/papaya.pdf>.

Van Droogenbroeck, B., T. Kybdt, I. Maertens, E. Romeijn-Peeters, X. Scheldman, J.P. RomeroMotochi, P. Van Damme, P. Goetghebeur, and G. Gheysen. 2004. Phylogenic analysis of the highland (Vasconcellea) and allied genera (Caricaceae) using PCR-RFLP. Theor. Appl. Genet. 108:1473-1486.

Wall, E.M., T.S. Lawrence, M.J. Green, and M.E. Rott. 2004. Detection and identification of transgenic virus resistant papaya and squash by multiplex PCR. Eur. Food Res. Technol. 219:90-96. 